\title{
The Ion-Matter Interaction with Swift Heavy Ions in the Light of Inelastic Thermal Spike Model
}

\author{
M. Toulemonde ${ }^{a}$, C. Dufour ${ }^{b}$ And E. PAumier ${ }^{b}$ \\ ${ }^{a}$ CIRIL, Laboratoire commun CEA-CNRS-ENSICAEN and Université de Caen \\ Bd H. Becquerel, BP 5133, 14070 Caen-Cedex 5, France \\ ${ }^{b}$ SIFCOM, Laboratoire commun CNRS, ENSICAEN and Université de Caen \\ 6 rue du Maréchal Juin, 14050 Caen-Cedex, France
}

\begin{abstract}
A description of the inelastic thermal spike model is presented in order to correlate the energy deposited by swift heavy ions to the nanometric matter transformation induced in inorganic metallic and insulating materials. Knowing that insulator is more sensitive than metallic material and that amorphous material is in general more sensitive than a crystalline one, it appears evident that the electron-phonon coupling constant $g$ plays a key role. It will be shown that in metallic material we are able to describe different phenomena with the same value of $g$ : for example, track formation with defect annealing or sputtering of atoms. In insulators the emphasis is made on results obtained for amorphizable materials like $\mathrm{SiO}_{2}$ quartz and for non-amorphizable ionic crystals like $\mathrm{CaF}_{2}$. Assuming that tracks result from a transient thermal process, a quantitative development of the model is proposed using the electron-atom mean free path $\lambda$ (inversely proportional to the square root of $g$ ) as a free parameter. With this parameter it is possible to quantitatively describe track radii in a wide range of ion velocities whatever the bonding character of the crystal is - assuming specific criteria: tracks may result from a rapid quenching of a cylinder of matter in which the energy deposited on the lattice has overcome either the energy necessary to reach a quasi-molten phase in the case of amorphizable materials or the vaporization energy in the case of non-amorphizable materials. The evolution of the $\lambda$ parameter of the considered insulator decreases versus the band gap energy. In this model, velocity effect, and a link between track formation and sputtering of atoms is established for amorphizable insulators while open questions appear for ionic crystals.
\end{abstract}

PACS numbers: 61.80.Az, 61.80.Jh

\section{Introduction}

The thermal spike effect $[1,2]$ has been always invoked to explain the track formation in insulators from the time when the first observations were made by 


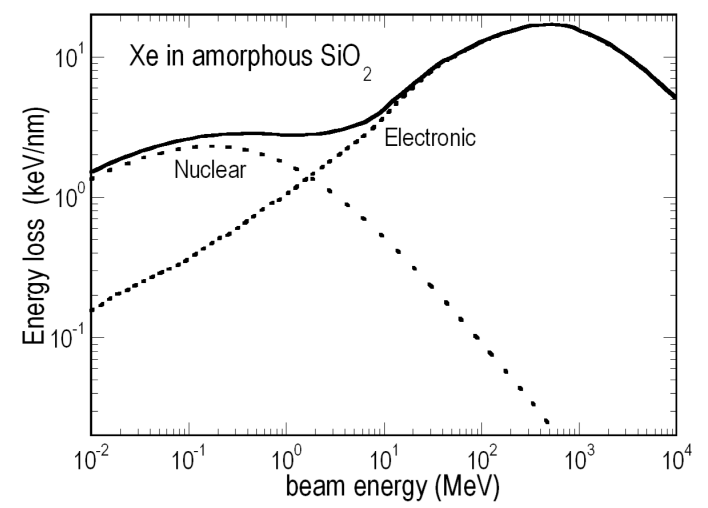

Fig. 1. Nuclear, electronic, and total energy loss versus beam energy for Xe ion in amorphous $\mathrm{SiO}_{2}$.

Silk and Barnes [3]. It is only since the 90's that this model [4-6] was developed in details to describe the damage induced in all kind of materials, metallic or insulator, irradiated by swift heavy ions. A typical evolution of the energy loss [7] of swift heavy ions as a function of the beam energy is presented in Fig. 1 and, for a typical beam of $\sim 370 \mathrm{MeV}$ Xe ion, the electronic energy loss is more than two orders of magnitude larger than the nuclear energy loss. When energetic ions slow down in solids, the energy is locally deposited to the electrons and finally can be transferred to the atoms by electron-electron and electron-atom interactions. When the energy deposited on the atoms overcomes the energy necessary to melt (defined as the energy to reach the melting temperature plus the enthalpy of fusion), the irradiated virgin materials are sensitive to the electronic energy loss $\left(S_{\mathrm{e}}\right)$ process. The main objective is to make quantitative link between macroscopic thermodynamical data — such as melting and vaporization energy — and sub-microscopic effects - the ion tracks. This correlation could be made despite the wide variety of ion track natures (point defects, defect annealing, crystallization, amorphization, phase change, sputtering,... ). It has been shown that metallic and insulating systems, whether they are amorphous or crystalline, respond to swift heavy ion irradiation according to a few relevant physical parameters among which the electron-phonon coupling plays a key role. The materials are consequently classified according to this parameter.

\section{The mathematical and physical hypothesis}

The matter is assumed to be composed of two sub-systems in interaction: the electrons described within the quasi free electron gas model and the atoms following the Debye model. Each sub-system is characterized by thermodynamic parameters: the temperature $T$, the specific heat $C$, the thermal conductivity $K$, and the latent heats of phase transformation (solid-liquid $\Delta H_{\mathrm{SL}}$, liquid--vapor $\Delta H_{\mathrm{LV}}$ and solid-vapor $\left.\Delta H_{\mathrm{SV}}\right)$ and the corresponding temperatures $T_{\mathrm{SL}}, T_{\mathrm{LV}}$, and $T_{\mathrm{SV}}$. The 
electronic and atomic temperature are governed by the following set of coupled equations that describe the thermal energy flows coming in and out of a cylindrical slice whose radius is comprised between $r$ and $r+\mathrm{d} r / 2$.

$$
\begin{aligned}
C_{\mathrm{e}} \frac{\partial T_{\mathrm{e}}(r, t)}{\partial t} & =-\frac{1}{c} \frac{\partial}{\partial r}\left(r K_{\mathrm{e}} \frac{\partial}{\partial r} T_{\mathrm{e}}(r, t)\right)-g\left(T_{\mathrm{e}}-T_{\mathrm{a}}\right)+A(r, t), \\
C_{\mathrm{a}} \frac{\partial T_{\mathrm{a}}(r, t)}{\partial t} & =-\frac{1}{r} \frac{\partial}{\partial r}\left(r K_{\mathrm{a}} \frac{\partial}{\partial r} T_{\mathrm{a}}(r, t)\right)+g\left(T_{\mathrm{e}}-T_{\mathrm{a}}\right) .
\end{aligned}
$$

The index e (resp. a) stands for electrons (resp. atoms). $g\left(T_{\mathrm{e}}-T_{a}\right)$ represents the so-called electron-phonon coupling which is the energy exchange between the electrons and the atoms.

In the following it will be shown how a complete numerical solution of these two equations can account for several physical observations. Such specific solution has proved to be necessary in order to account the initial energy distribution $A(r, t)$ determined from Monte-Carlo calculations [8], and the evolution of all the parameters of the two subsystems (electron and atoms) with the temperature. The aim of the calculation is to evaluate the radius in which the deposited energy has to overcome the melting energy $E_{\mathrm{F}}$ (the energy to reach the melting temperature from the irradiation temperature plus the enthalpy of fusion) or even the vaporizing energy $E_{\mathrm{v}}\left(E_{\mathrm{F}}\right.$ plus the energy to reach the vaporization temperature plus the enthalpy of vaporization).

\section{Metallic materials}

\subsection{Defect annealing}

The irradiation effects produced by swift heavy ions in iron have been evidenced by Dunlop et al. [9]. The number of created defects was determined [4, 9] by measuring in situ the resistivity increment $\Delta \rho$ as a function of the ion fluence $\Phi \times t$, where $\Phi$ is the flux of incident particle and $t$ - the time of irradiation. Such a number, normalized by the theoretical number of defects created by nuclear collisions (TRIM cascade [7]), is defined as the damage efficiency. For example for iron (Fig. 2), the damage efficiency decreases till a value of $S_{\mathrm{e}}$ equal to $38 \mathrm{keV} / \mathrm{nm}$, supporting the idea of a defect annealing, and then increases significantly indicating that a new phenomena of damage creation results from the electronic excitation.

Assuming that such a damage annealing is a thermal process, it has been investigated in the frame of the inelastic thermal spike (i-TS) model in metals. Knowing the evolution of $T_{\mathrm{a}}(r, t)$, the thermal atomic jumps in solid have been estimated [10] using the following relation:

$$
\begin{aligned}
& N_{\mathrm{s}}(r)=N_{\mathrm{i}}(r) \prod_{t=0}^{\infty}\left[1-\nu\left(T_{\mathrm{a}}(r, t) \Delta t\right)\right], \\
& \nu\left(T_{\mathrm{a}}(r, t)\right)=\frac{k T_{\mathrm{D}}}{h} \exp \left(-\frac{E_{\mathrm{a}}}{k T_{\mathrm{a}}(r, t)}\right),
\end{aligned}
$$

$N_{\mathrm{i}}(r)$ is the initial radial distribution of defects created by nuclear collisions, $N_{\mathrm{s}}(r)$ 


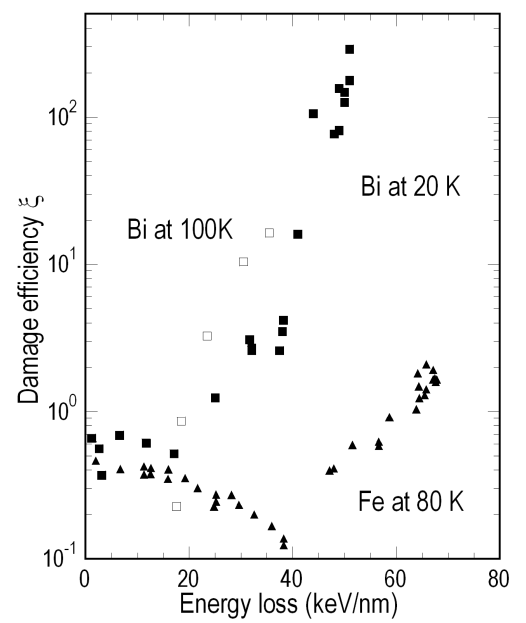

Fig. 2. Damage efficiency $\xi$ versus energy loss for Fe irradiated at $80 \mathrm{~K}$ [9], and Bi irradiated at $20 \mathrm{~K} \mathrm{[4]}$ and at $100 \mathrm{~K}$ [11].

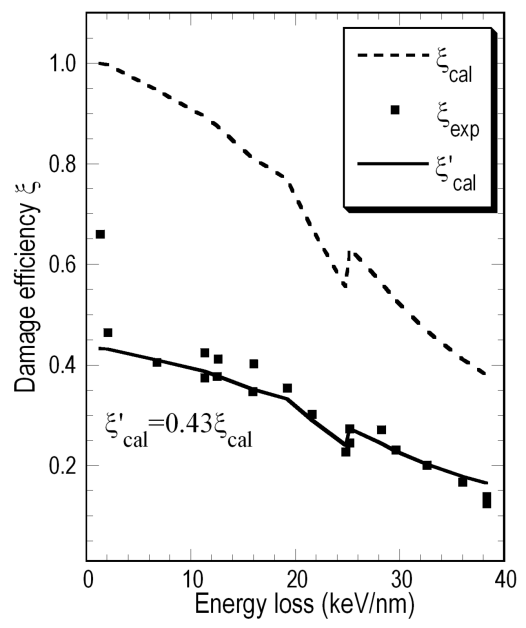

Fig. 3. Damage efficiency in Fe [9], compared with theoretical approach in the i-TS model [10]. The factor of 0.43 , used in order to compare directly the results of the calculations to the experiments, can be understood as a possible a-thermal annealing, known to exist in the nuclear collision regime.

is the remaining defects after the irradiation, $\nu\left(T_{\mathrm{a}}(r, t)\right)$ is the probability to anneal a defect in a cylinder shell at a distance $r$, at a temperature $T_{\mathrm{a}}(r, t)$ and at a time $t, k$ is the Boltzmann constant, $h$ - the Planck constant, $T_{\mathrm{D}}$ - the Debye temperature, and $E_{\mathrm{a}}$ — the annealing activation energy of the considered defect.

Determining the spatial distributions vacancies and interstitials created by nuclear collisions [10], the rate of recombination of existing defects for given swift 
heavy ion irradiation have been determined by adjusting the value of the electronphonon coupling to $1.44 \times 10^{12} \mathrm{~W} / \mathrm{cm}^{3} / \mathrm{s}$, in accord with the one that we can deduce from phenomenological approaches [5]. In agreement with experiments, the calculations can predict that the significant defect annealing appears for $S_{\mathrm{e}}$ values between 15 and $38 \mathrm{keV} / \mathrm{nm}$ (Fig. 3).

With the same value of the electron-phonon coupling, extrapolation to higher

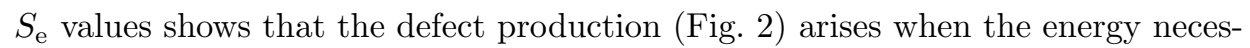
sary to melt is reached. By comparison to the damage efficiencies, our numerical calculations can describe, by a transient thermal process, defect annealing, and defect production in pure metal.

\subsection{Temperature of irradiation: application to Bi}

Bismuth $[4,11]$ material was especially experimentally studied to be compared with the inelastic thermal spike model due to its low value of energy to melt ( $\sim 0.38 \mathrm{eV} /$ atom as compared to $\sim 0.75 \mathrm{eV} /$ atom for Fe). As done for the iron, the rate of damage creation in $\mathrm{Bi}$, irradiated at two different temperatures, $20 \mathrm{~K}$ and $100 \mathrm{~K}$, was determined by measuring the resistivity increment $\Delta \rho$ in situ as a function of the ion fluence $\Phi \times t$. Applying also the same analysis, the damage efficiencies were plotted versus $S_{\mathrm{e}}$ (Fig. 2). Firstly, it is clear that the $S_{\mathrm{e}}$ threshold of damage efficiency is lower for $\mathrm{Bi}$ than for $\mathrm{Fe}$, in agreement with the fact that the energy necessary to melt is lower in Bi than in Fe. Secondly, the damage efficiency increases when going from $20 \mathrm{~K}$ to $100 \mathrm{~K}$ as expected in the frame of the i-TS model since the energy necessary to melt decreases when increasing the temperature of irradiated sample [11].

\subsection{Track radii}

A specific analysis of $\Delta \rho\left(\Phi_{t}\right)$ curves [4], using a statistical approach [12], allows us to deduce the cylinder radius in which the defects are created (Fig. 4). Assuming that these defects result from the quenching of a molten phase along the ion path (i.e. the deposited energy on the atoms is larger than $E_{\mathrm{F}}$ ), it is possible to predict the evolution of the track radii in different materials: crystalline iron [9], amorphous iron boron alloy [13], and $\mathrm{Bi}[4,11]$. In Bi large radii can be reached because, with its low value of the electron-phonon (e-ph) coupling $\left(g \sim 1.4 \times 10^{11} \mathrm{~W} / \mathrm{cm}^{3} / \mathrm{s}\right)$, the energy deposited on the electrons diffuses on a large distance before a transfer to the atoms. The same prediction is done for tracks created in Fe, using the e-ph coupling $\left(g=1.44 \times 10^{12} \mathrm{~W} / \mathrm{cm}^{3} / \mathrm{s}\right)$ determined by the defects annealing. Now going to amorphous materials, it is known that e-ph coupling constant increases as compared to a crystalline material and then, according to this model, they should be more sensitive than the same material in its crystalline phase. Experimentally, it is true since the track radii are larger in an amorphous metallic alloys $\left(\mathrm{a}-\mathrm{Fe}_{85} \mathrm{~B}_{15}\right)$ than in a crystalline Fe (Fig. 4). Then increasing the e-ph coupling to $5 \times 10^{12} \mathrm{~W} / \mathrm{cm}^{3} / \mathrm{s}$, one can reproduce the experimental track radii (Fig. 4) in an amorphous metallic alloys. 


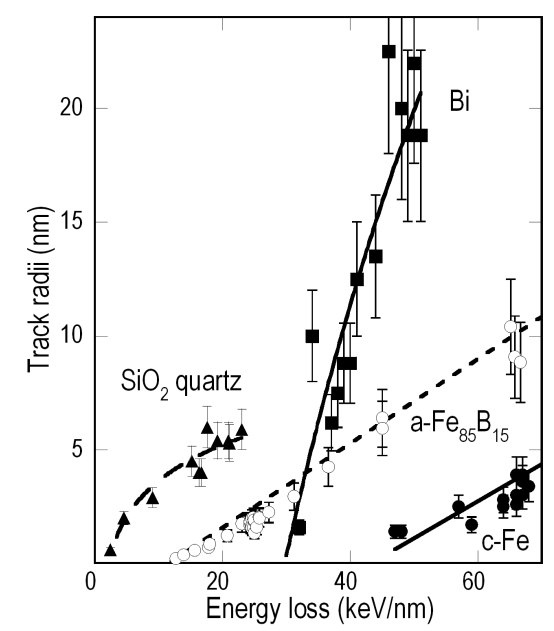

Fig. 4. Track radii versus energy loss in crystalline $\mathrm{Fe}$ (c-Fe) [9], in amorphous a- $\mathrm{Fe}_{85} \mathrm{~B}_{15}$ [13], in $\mathrm{Bi}[4,11]$, and in $\mathrm{SiO}_{2}$ quartz (crystalline material) [19].

\subsection{Atoms sputtering in $\mathrm{Ti}$}

In the framework of the inelastic thermal spike model, the total yield $Y_{\text {tot }}$ of particles evaporated from the surface is determined from the time and space integral of the local evaporation rate $Y_{\text {tot }}(r)$ as a function of the local temperature:

$$
\begin{aligned}
& T_{\text {tot }}=\int_{0}^{\infty} \mathrm{d} t \int \Phi\left(T_{\mathrm{a}}(r, t)\right) 2 \pi r \mathrm{~d} r, \\
& \Phi\left(T_{\mathrm{a}}(r, t)\right)=N \sqrt{\frac{k T_{\mathrm{a}}(t, r)}{2 \pi M}} \exp \left(\frac{-U}{k T_{\mathrm{a}}(t, r)}\right),
\end{aligned}
$$

where $N$ is the atomic density, $k$ is the Boltzmann constant and $M$ is the molecular mass of the target atom. The surface binding energy $U$ is assumed to be equal to the sublimation energy per evaporated molecule.

Sputtering yields and typical angular distributions of pure metals were measured with heavy ions at energies where electronic energy loss dominates [14]. Using different ion charge states and ion/energy combinations, electronic effects were clearly observed in sputtering of metals such as $\mathrm{Ti}$ and $\mathrm{Zr}$, but not in Au. Angular distributions and total yields of atoms sputtered from Ti, Zr, and Au targets are measured and follow an overcosine law $\left(\cos ^{3} \theta\right)$. By assuming an azimuthal symmetry, the total yield can be deduced from the measured angular distribution.

By applying the pure inelastic thermal spike model, it was not possible to reproduce the measured yields (Fig. 5, calculations made with $1.5 \mathrm{MeV} / \mathrm{u}$ ion energy compared to I and $\mathrm{Au}$ irradiations at equivalent energies). In addition to the electronic inelastic collisions, elastic collision nuclear spike effects had to be included assuming a superheating scenario [15]. The results for Ti (Fig. 5) demonstrate the importance of the synergy of both heating mechanisms (electronic + nuclear) for sputtering in this energy regime in metallic materials. 


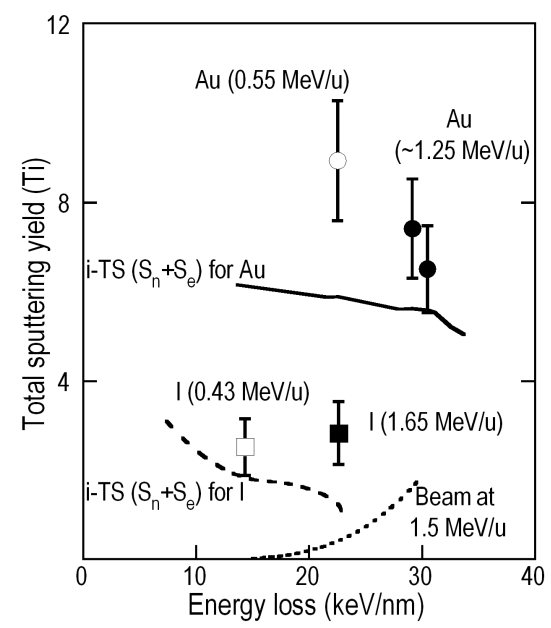

Fig. 5. Sputtering yield of Ti irradiated by ${ }^{127} \mathrm{I}$ (squares) and ${ }^{197} \mathrm{Au}$ (circles) ions at different energies quoted in the picture. Calculations were performed in the case of a pure electronic effect (curve labelled "beam at $1.5 \mathrm{MeV} / \mathrm{u}$ ") and with a synergy between nuclear and electronic energy losses (curve labelled i-TS $\left(S_{\mathrm{e}}+S_{n}\right)$ for I or Au).

\section{Insulators: electron mean free path, track radii, atom sputtering, and velocity effect}

Regarding the tracks observed by transmission electron microscopy [16, 17], the insulating materials are classified among amorphizable materials [18] such as $\mathrm{SiO}_{2}$ [19] or non-amorphizable ones such as $\mathrm{SnO}_{2}[20]$ and $\mathrm{CaF}_{2}[6,17]$. The only free parameter is the electron-lattice interaction mean free path $\lambda$ which is directly inversely linked to e-ph coupling constant $g$ by $\lambda^{2}=D_{\mathrm{e}} C_{\mathrm{e}} / g$ relation $\left(D_{\mathrm{e}}\right.$ - electronic diffusivity, $C_{\mathrm{e}}$ - electronic specific heat).

With this parameter, whatever is the bonding character of the crystal, it is possible to evaluate the track radii, assuming the tracks may result from a rapid quenching of a cylinder of matter in which the energy deposited on the lattice has overcome either the energy necessary to reach a quasi-molten phase $\left(E_{\mathrm{F}}\right)$ in the case of amorphizable materials or the vaporization energy $\left(E_{\mathrm{v}}\right)$ in the case of non-amorphizable materials [6]. Such assumptions are illustrated in Fig. 6, showing that the best fit of track data, using lambda as a free parameter, is obtained with the following assumptions, melt phase for the amorphizable $\mathrm{SiO}_{2}$ quartz and vapor phase for the ionic crystal of $\mathrm{CaF}_{2}$. Applying such fits and hypothesis on different materials, the evolution of the $\lambda$ parameter versus the band gap energy of the considered insulator is presented in Fig. 7. Such a picture is in agreement with the fact that the higher is the band gap energy, the larger should be the electronphonon coupling, i.e. the smaller the electron mean fee path $\lambda[6]$.

To ensure the previous correlation, one has always to take into account an important parameter which is the energy density deposited on the electrons that 


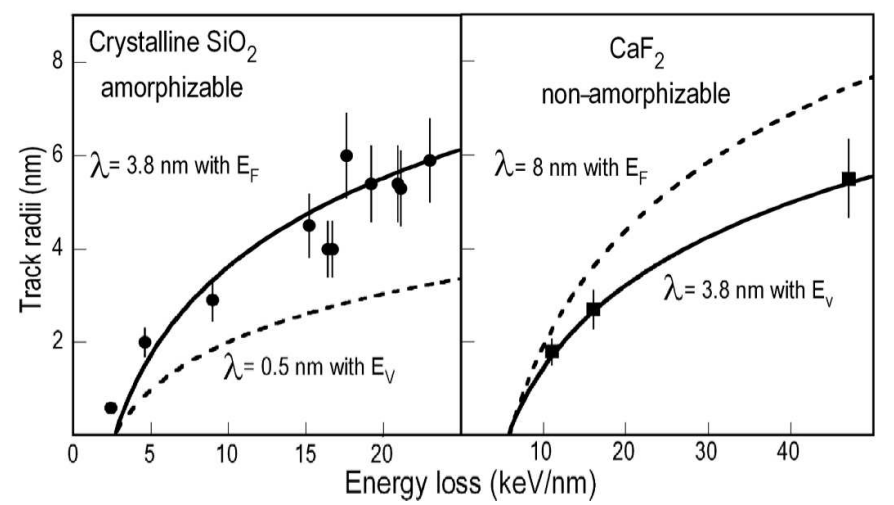

Fig. 6. Track radii versus energy loss for the amorphizable $\mathrm{SiO}_{2}$ quartz (left) and for the non-amorphizable ionic $\mathrm{CaF}_{2}$ crystal (right). Using the two criteria, either the energy necessary to melt $\left(E_{\mathrm{F}}\right)$ or to vaporize $\left(E_{\mathrm{v}}\right)$, and adjusting the $\lambda$ value to the threshold of damage creation [6], the best fit for $\mathrm{SiO}_{2}$ is obtained by assuming the energy necessary to melt for the $\mathrm{SiO}_{2}$ quartz whereas it is needed to assume the energy necessary to vaporize to reproduce the track in $\mathrm{CaF}_{2}$.

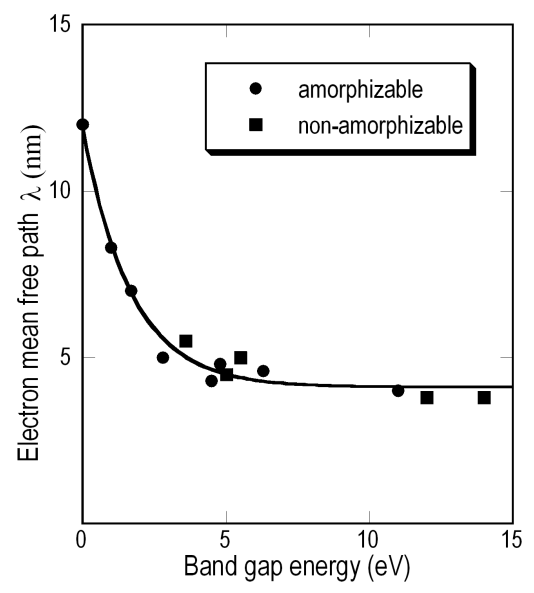

Fig. 7. Electron mean free path versus band gap energy for different insulators amorphizable or non-amorphizable [6].

varies with the ion velocity [8]. In Fig. 1, the same value of the energy loss can be reached for two different values of the beam energy. A detailed study of this effect has been done on yttrium iron garnet (Fig. 8) [21, 22]. Using the results of the Monte-Carlo calculations [8] that defined the radial expansion of the energy deposited on the electrons, the track radii in yttrium iron garnet can be fitted with the same $\lambda(=5 \mathrm{~nm})$ value independently of the beam energy.

In this frame, the sputtering should be correlated to an evaporation process. Assuming a superheating scenario as for metallic materials, it is possible to predict 


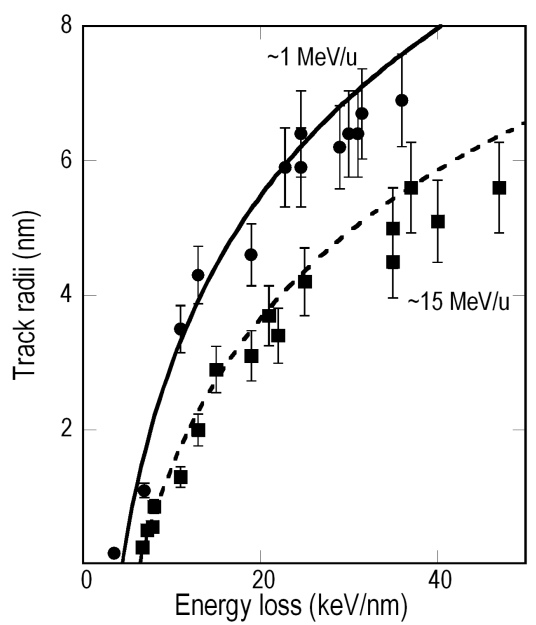

Fig. 8. Track radii versus energy loss for two different ranges of beam energy irradiation $(\sim 1 \mathrm{MeV} / \mathrm{u}$ and $\sim 15 \mathrm{MeV} / \mathrm{u})$. The lines are the result of the i-TS model calculation using a unique value of the electron mean free path.

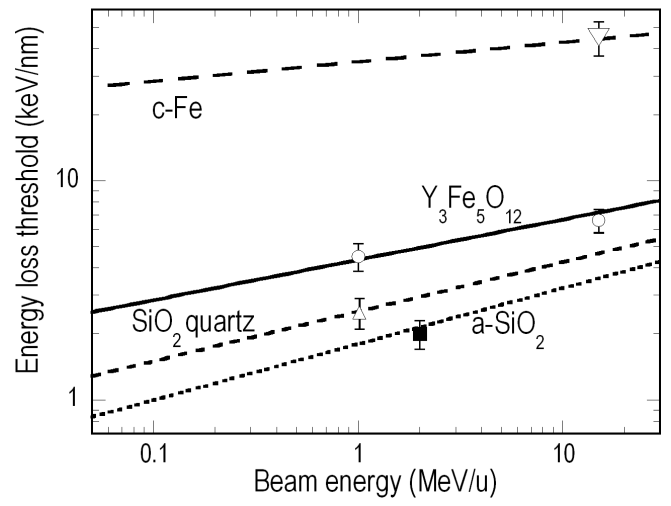

Fig. 9. Energy loss threshold of damage creation in different materials (crystalline iron, yttrium iron garnet $\left(\mathrm{Y}_{3} \mathrm{Fe}_{5} \mathrm{O}_{12}\right)$, crystalline $\mathrm{SiO}_{2}$ quartz and amorphous a-SiO 2 ) versus beam energy.

the track radii and the sputtering rate with the same lambda value [23] for $\mathrm{SiO}_{2}$ quartz (Fig. 9). However, sputtering measurements show new phenomena like a jet-like component in ionic crystal associated with a huge sputtering [23] that cannot be explained in the frame of the thermal spike model.

\section{Perspective aspects and conclusions}

A good correlation has been found between theoretical results and experimental data provided that the only free parameter of the model should be known: the electron-phonon coupling constant or the electron mean free path. In metallic 
materials, this relationship between the behavior of metals under irradiation with swift heavy ions and the electron-phonon coupling strength $g$ is performed by assuming the track results from the quenching of a cylinder of matter in which the energy has overcome $E_{\mathrm{F}}$, the energy necessary to melt the material along the ion path. It is more complicated for insulators where the correlation with the electron mean free path is achieved using two specific criteria: either the energy necessary to melt for an amorphizable material or the energy necessary to vaporize in a non-amorphizable ionic materials.

Such agreement allows us to predict the evolution of the electronic threshold of damage creation by electronic energy losses for different materials versus beam energy (Fig. 10). It is shown that it is not always necessary to have high energy beam to induce damage by electronic excitation. For example, the anisotropic growth [24] - a specific phenomenon that appears in amorphous materials only in the electronic excitation [25] regime - is still observable with $0.3 \mathrm{MeV}$ of $\mathrm{Xe}$ ion. It is also shown that amorphous material (Figs. 4 and 10) is always more sensitive than crystalline one whatever the material is - a metal or an insulator.

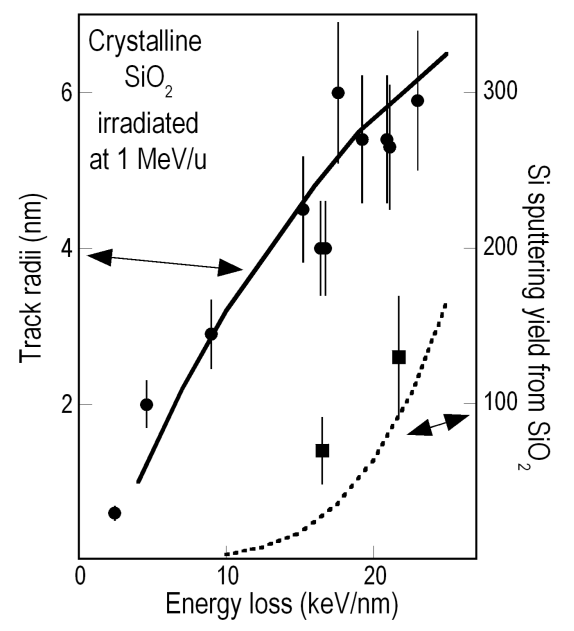

Fig. 10. Calculations of the track radii and the sputtering yield in crystalline $\mathrm{SiO}_{2}$ quartz with the same value of the electron mean free path.

Ionic crystals seem rather more complicated to be explained in the framework of the i-TS model: what kind of track could arise from the quenching of the melt phase created along the ion path (since the observable track by electron microscopy results from the quench of a "vapor" phase) and why do we have a huge sputtering yield (more than 10000 per incident ions in $\mathrm{LiF}$ as compared to 150 for $\mathrm{SiO}_{2}$ quartz [23])? The answer to these questions can be a severe test of the inelastic thermal spike model.

The overall agreement between experimental results and the i-TS model should not hide the hypothesis made in the calculations [1, 2, 4-6]: a transient 
thermal process at short time with parameters measured at equilibrium. However, the weakness of the hypothesis (in bulk melting and vapor phases as criteria) should not prevent to develop this model in order to touch its limits.

\section{References}

[1] I.M. Lifshitz, M.I. Kaganov, L.V. Taratanov, J. Nucl. Energy A 12, 69 (1960).

[2] L.T. Chadderton, H.M. Montagu-Pollock, Proc. R. Soc. Lond. A 274, 239 (1986).

[3] E.C.H. Silk, R.S. Barnes, Philos. Mag. 4, 970 (1959).

[4] C. Dufour, A. Audouard, F. Beuneu, J. Dural, J.P. Girard, A. Hairie, M. Levalois, E. Paumier, M. Toulemonde, J. Phys., Condens. Matter 5, 4573 (1993).

[5] Z.G. Wang, Ch. Dufour, E. Paumier, M. Toulemonde, J. Phys. Condens. Matter 6, 6733 (1994) and J. Phys. Condens. Matter 7, 2525 (1995).

[6] M. Toulemonde, C. Dufour, A. Meftah, E. Paumier, Nucl. Instrum. Methods B 166-167, 903 (2000).

[7] J.P. Biersack, L.G. Haggmark, Nucl. Instrum. Methods 174, 257 (1980).

[8] M.P.R. Waligorski, R.N. Hamm, R. Katz, Nucl. Tracks Radiat. Meas. 11, 3 (1986).

[9] A. Dunlop, D. Lesueur, P. Legrand, H. Dammak, J. Dural, Nucl. Instrum. Methods, Phys. Res. B 90, 330 (1994).

[10] Z.G. Wang, C. Dufour, E. Paumier, M. Toulemonde, Nucl. Instrum. Methods B 115, 577 (1996).

[11] C. Dufour, F. Beuneu, E. Paumier, M. Toulemonde, Euro Phys. Lett. 45, 585 (1999).

[12] P. Thévenard, G. Guiraud, C.H.S. Dupuy, B. Delaunay, Radiat. Eff. 32, 83 (1977).

[13] A. Audouard, E. Balanzat, G. Fuchs, J.C. Jousset, D. Lesueur, L. Thomé, Europhys. Lett. 3, 327 (1987) and J. Phys., Condens. Matter 5, 995 (1993).

[14] H.D. Mieskes, PhD Thesis, LMU, München 1999.

[15] H.D. Mieskes, W. Assmann, F. Grüner, H. Kucal, Z.G. Wang, M. Toulemonde, Phys. Rev. B 67, 155414 (2003).

[16] F. Studer, M. Hervieu, J.M. Costantini, M. Toulemonde, Nucl. Instrum. Methods B 122, 449 (1997).

[17] J. Jensen, A. Dunlop, S. Della Negra, Nucl. Instrum. Methods B 141, 753 (1998).

[18] A. Meftah, J.M. Costantini, N. Khalfaoui, S. Boudjadar, J.P. Stoquert, F. Studer, M. Toulemonde, Nucl. Instrum. Methods B 237, 563 (2005).

[19] A. Meftah, F. Brisard, J.M. Costantini, E. Dooryhee, M. Hage-Ali, M. Hervieu, J.P. Stoquert, F. Studer, M. Toulemonde, Phys. Rev. B 49, 12457 (1994).

[20] A. Berthelot, S. Hemon, F. Gourbilleau, C. Dufour, E. Dooryhee, E. Paumier, Nucl. Instrum. Methods B 146, 437 (1998) and Philos. Mag. A 80, 2257 (2000).

[21] A. Meftah, F. Brisard, J.M. Costantini, M. Hage-Ali, J.P. Stoquert, F. Studer, M. Toulemonde, Phys. Rev. B 48, 920 (1993).

[22] A. Meftah, J.M. Costantini, M. Djebara, N. Khakfaoui, J.P. Stoquert, F. Studer, M. Toulemonde, Nucl. Instrum. Methods B 122, 470 (1997). 
[23] M. Toulemonde, W. Assmann, F. Grüner, C. Trautmann, Phys. Rev. Lett. 88, 057602 (2002).

[24] T. van Dillen, A. Polman, C.M. van Kats, A. von Bladereen, Appl. Phys. Lett. 83, 4315 (2003).

[25] S. Klaumünzer, Hou Ming-Dong, G. Schumacher, Phys. Rev. Lett. 57, 850 (1986). 